\title{
Brief Report: The Social Responsiveness Scale for Adults (SRS-A): Initial Results in a German Cohort
}

\author{
Sven Bölte
}

Published online: 20 December 2011

(C) The Author(s) 2011. This article is published with open access at Springerlink.com

\begin{abstract}
The Social Responsiveness Scale (SRS) is a tool for quantitative autism assessment in children and adolescents. The SRS-A addresses social responsiveness in adulthood. Reliability and validity using the German adaptation of the SRS-A was examined in 20 adults with Autism Spectrum Disorder (ASD), 62 with other mental disorders (CLIN) and 163 typically developing (TD) participants. Cronbach's alpha ranged from .71 (TD) to .89 (ASD). A SRS-A total score of 67 had a sensitivity of .85 , and a specificity of .83 for ASD versus CLIN/TD. Correlations with established autism scales (ADOS, AQ, SCQ) were moderate to high $(r=.25-.83)$. Results provide adequate preliminary support for the application of the SRS-A.
\end{abstract}

Keywords Questionnaire - Broader phenotype ·

Psychometrics · Screening · Assessment

The Social Responsiveness Scale (SRS; Constantino et al. 2003) is a quantitative measure of autistic traits in 4-18 year olds. The SRS has demonstrated good psychometric properties and cross-cultural validity for Autism Spectrum Disorder (ASD) assessment (Bölte et al. 2008, 2011). A revised form extended the application of the SRS with items modified to address social responsiveness in adulthood (SRS-A; Constantino and Todd 2005). Like the child version, the SRS-A contains 65 Likert-scaled (0-3) items,

\footnotetext{
S. Bölte $(\bowtie)$

Department of Women's and Children's Health, Center of Neurodevelopmental Disorders at Karolinska Institutet (KIND), Karolinska Institutet, Astrid Lindgren Children's Hospital, Q2:07, 17176 Stockholm, Sweden

e-mail: sven.bolte@ki.se
}

generates a singular scale with a maximum score of 195 for behavior shown in the last 6 months, and takes 15-20 min to complete. Possible administrations are self-, parent-, other relative-, and spouse-report. Yet, only sparse information has been published on this instrument (Virkud et al. 2009). The present study, using a German adaptation, provides a range of new results on the SRS-A.

Three samples were collected: First, parent reports at a specialized autism center on $N=20$ ( 5 females, 15 males) individuals with high functioning ASD aged 18-36 years with a mean age of 27.5 years $(S D=6.5)$, and a mean full scale IQ on the Wechsler Intelligence Scales for Adults of 112.8 (SD = 16.4). Single clinical diagnoses of 10 Asperger's syndrome, 5 autism, and 5 atypical/PDD-NOS were based on ICD-10 research criteria, corroborated by findings from the Autism Diagnostic Interview-Revised and Autism Diagnostic Observation Schedule (ADOS). Second, spouse-report on a mixed adult psychiatric sample (non-ASD) (CLIN) of $N=62$ individuals (28 females, 34 males) aged $28-56$ years $(M=40.0, \mathrm{SD}=6.6)$. Third, SRS-A data on $N=163$ (89 females, 74 males) typically developing adults (TD) aged 19-79 years with reporting sources varied among spouses, siblings, and others.

Raw scores/discriminant validity. The SRS-A mean total score was highest in the ASD sample $(M=78.5, \mathrm{SD}=$ 13.7), intermediate in the CLIN $(M=63.4, \mathrm{SD}=15.4)$, and lowest in the $\mathrm{TD}(M=55.5, \mathrm{SD}=9.9)\left(F_{2 / 244}=\right.$ 36.8, $p<.0001$, partial $e t a^{2}=.24,1-\beta$ observed $=$ 1.00). Receiver operating characteristics (ROC) analyses in 20 ASD versus 245 CLIN plus TD cases showed an area under the curve of AUC $=.88(p<.001)$ (Fig. 1), with a SRS-A total score of 67 showing a balance of sensitivity (.85) and a specificity (.83) for ASD. Internal consistency. Cronbach's alpha was .89 in the ASD, .84 in CLIN, and .71 in the TD. Concurrent validity. Availability of other 


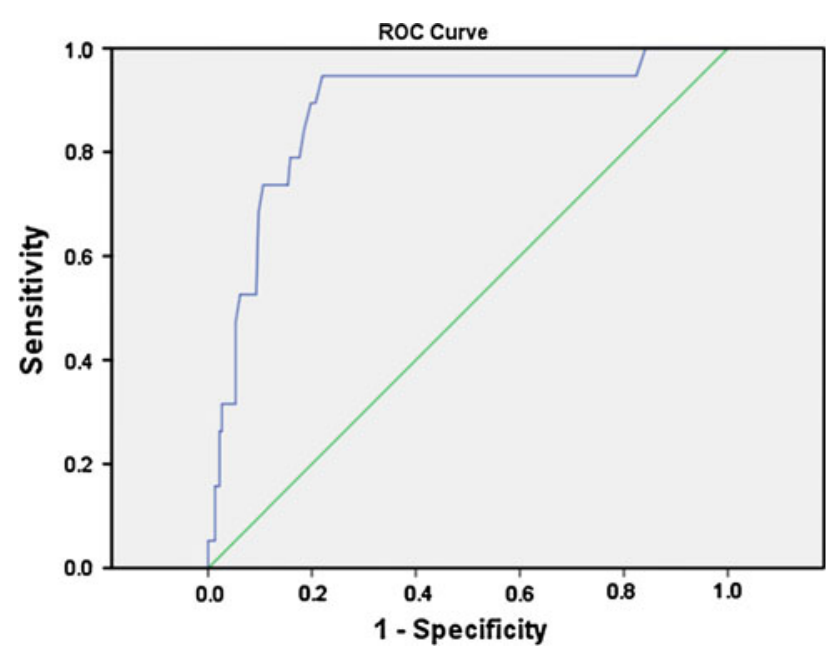

Fig. 1 Receiver operating characteristics (ROC) curve of the Social Responsiveness Scale for Adults (SRS-A) for the condition ASD $(n=20)$ versus TYP and CLIN $(n=245)$; area under the curve $=.88(p<.001)$

measures was limited to the ASD group. Within this group, correlations of the SRS-A total score were: $r$ (Pearson) $=$ $.83(p<.001)$ with the Social Communication Questionnaire (SCQ) total score (lifetime version), $r=.25$ $(p=.09)$ with the ADOS module 4 social-communication algorithm subscale score, and $r=.35(p=.04)$ the Autism Spectrum Quotient (AQ) total score.

The findings reported here provide adequate preliminary support for the application of the SRS-A for the assessment of autism traits in adults. These findings parallel those seen in the extensive literature on the standard child SRS. It may be noted that the actual spread between TD and ASD on the SRS-A total score (55.5 vs. 78.5 ) is not as wide as has been found for the standard SRS (e.g. $\sim 24$ vs. $\sim 102$; Bölte et al. 2008, in German samples). The latter might be partially explained by the fact that the ASD group included mostly high functioning adults showing less extreme phenotypic expression of autistic symptoms than would be typical for the spectrum as a whole. In addition, rater type (mother, father, spouse, sibling, others) might be crucial for SRS-A information, and was not comparable between samples in this study. Thus, it is clear that replication and extension into more widely varied and larger samples is required to further endorse the utility of the SRS-A. Furthermore, replication is needed for the original SRS-A, and other adaptations, as the present results might be specific to a German sample and translation.

Conflict of interest The author of this article disclose the following potential conflicts of interests: Sven Bölte receives royalties for the German version of the Social Responsiveness Scale ("Skala zur Erfassung sozialer Reaktivität') from Hans Huber Publishers.

Open Access This article is distributed under the terms of the Creative Commons Attribution Noncommercial License which permits any noncommercial use, distribution, and reproduction in any medium, provided the original author(s) and source are credited.

\section{References}

Bölte, S., Poustka, F., \& Constantino, J. N. (2008). Assessing autistic traits: cross-cultural validation of the Social Responsiveness Scale (SRS). Autism Research, 1, 354-363.

Bölte, S., Westerwald, E., Holtmann, M., Freitag, C., \& Poustka, F. (2011). Autistic traits and autism spectrum disorders: The clinical validity of two measures presuming a continuum of social communication skills. Journal of Autism and Developmental Disorders, 41, 66-72.

Constantino, J. N., \& Todd, R. D. (2005). Intergenerational transmission of subthreshold autistic traits in the general population. Biological Psychiatry, 57, 655-660.

Constantino, J. N., Davis, S., Todd, R., Schindler, M., Gross, M., Brophy, S., et al. (2003). Validation of a brief quantitative measure of autistic traits: Comparison of the Social Responsiveness Scale with the Autism Diagnostic Interview-Revised. Journal of Autism and Developmental Disorders, 33, 427-433.

Virkud, Y. V., Todd, R. D., Abbacchi, A. M., Zhang, Y., \& Constantino, J. N. (2009). Familial aggregation of quantitative autistic traits in multiplex versus simplex autism. American Journal of Medical Genetics B Neuropsychiatric Genetics, 150B, $328-334$. 